BIOMEDICAL AND BIOSOCIAL ANTHROPOLOGY
$\begin{gathered}\text { Official Journal of the International Academy } \\ \text { of Integrative Anthropology } \\ \text { journal homepage: http://bba-journal.com }\end{gathered}$

\title{
Determination of cephalometric parameters, which usually do not change during surgical and orthodontic treatment depending on facial types according to Garson in Ukrainian young men and young women with orthognathic occlusion
}

\author{
Drachevska I. Yu. ${ }^{1}$, Dmitriev M. 0. ${ }^{1}$, Perera Clifford ${ }^{2}$, Shevchenko V. M. ${ }^{1}$, Gunas I. V. ${ }^{1}$ \\ ${ }^{1}$ National Pirogov Memorial Medical University, Vinnytsya, Ukraine
} 2University of Ruhuna, Matara, Sri Lanka

\section{ARTICLE INFO}

Received: 27 October 2020

Accepted: 27 November 2020

UDC: $616.314 .2-007.271-$

053.7:617.52:616-073.75

\section{CORRESPONDING AUTHOR}

e-mail: idra4evskaya@gmail.com Drachevska I. Yu.

\begin{abstract}
Aesthetics is one of the key elements that accompanies modern medicine, in particular, dentistry. The need for treatment not only eliminates the symptoms of the disease but also leads to the restoration or improvement of the aesthetic appearance of the person, and especially the face, gave impetus to the development of new areas of science and technology. An important step in the implementation of such treatments is experimental research to identify the norm for certain categories of the population, primarily according to sex and ethnic group. The aim of the study was to establish the characteristics of cephalometric parameters in Ukrainian young men and young women with orthognathic occlusion, which usually do not change during surgical and orthodontic treatment, depending on sex and facial types according to Garson. On the basis of the clinic "Vinintermed" teleroentgenography was performed using a dental cone-beam tomograph Veraviewepocs $3 D$ Morita (Japan). Cephalometric examination of lateral teleroentgenograms in 46 young men (aged 17 to 21 years) and 72 young women (aged 16 to 20 years) with orthognathic occlusion was done. The type of face was determined according to the morphological index of Garson. The evaluation of parameters that usually do not change during surgical and orthodontic treatment was performed in the licensed package "Statistica 6.0" using non-parametric methods of evaluation of the results. The percentile ranges of cephalometric parameters, which usually do not change during surgical and orthodontic treatment depending on the facial types of Ukrainian young men and young women with orthognathic occlusion has been established. Pronounced manifestations of sexual dimorphism of cephalometric parameters, which usually do not change during surgical and orthodontic treatment (higher values in young men) are established only for most linear dimensions (values of distances NS, Ar-Go, N-Se, N-CC, P-PTV and S-Ar) in representatives of different types of faces. Both between young men and young women with different face types, there are almost no reliable or tendencies of differences in cephalometric parameters, which usually do not change during surgical and orthodontic treatment.

Keywords: teleroentgenography, cephalometry, young men, young women, face types, orthognathic occlusion.
\end{abstract}

\section{Introduction}

One of the key issues in orthodontics, which has become relevant in recent decades, is the study of the variability of facial features, skull structure and their relationship to the position of the teeth among members of a particular ethnic group.

Thus, researchers working in this direction are already aware of the existence of works where the data on the specificity of the above indicators among different nationalities are confirmed. 127 ethnic Moroccans underwent teleroentgenography to identify the relationships between cephalometric and odontometric parameters. Statistical analysis of the obtained data revealed the existence of correlations between the upper posterior alveolar height and the anterior height of the face, the 
posterior height of the face. The lower anterior alveolar height at the same time is negatively correlated with the face height index [1].

For residents of western Ukraine, the existence of reliable correlations of medium strength between the linear dimensions of molars (parietal-lingual and mesio-distal dimensions) and indicators of the skull. With the indicators of the facial skull, the largest number of reliable and medium correlations with the height of teeth, crowns, root length, parietal-lingual and mesio-distal dimensions of molars [12]. A similar study, however, conducted with the population of southern Ukraine, found correlations between most sizes of molars on the lower jaw and the length and height of the nose [24].

Indian scientists have identified the size of the brain and facial skull for residents of Gujarat. The control group was residents of other Indian states. It was found that the standard deviation of the main and front index for residents of the state was 5.3345 and 6.0040 , and for residents of other states -7.3472 and 6.0525 , respectively. Most people in Gujarat were mesocephalic and dolichocephalic, while people in other states were dolichocephalic and brachycephalic [22].

Manifestations of sexual dimorphism in the features of cephalometric and odontometric indicators were revealed for the people of Korea. The height of the anterior alveolar process of the upper jaw correlated with the deviation of the upper incisor; moreover, higher power correlations were found in women compared to men [13].

A comparative analysis of cephalometric and odontometric parameters between the populations of Japan and Bangladesh found that the inhabitants of the latter are characterized by lower face height $(p<0.01)$, higher indicators of the position of the upper and lower jaws $(p<0.01)$ compared with the population of Japan [2].

In another study, an analysis of data from mainland Japan and the Ryukyu Islands found that women from the island of Japan had smaller mandibular incisor, less depth of the upper lip, and an inclined to the front mandibular symphysis [26].

In addition to identifying the ethnic characteristics of odontometric and cephalometric indicators, an important component of the study is to identify the overall relationships and effects of one component on another and vice versa, the strength of these relationships and their direction, etc. $[7,8]$.

A group of researchers from Ukraine and India [10] by regression analysis built reliable models to determine the characteristics of the position of the central incisors of the upper and lower jaw according to the Steiner method. The study confirmed the existence of a relationship between the angle ANB and the angles Max1_NA and Max1_SN and the distance 1u_NA.

However, it is also worth paying attention to the method of obtaining data on the features of cephalometric parameters of both the cranial and facial parts of the skull.
Thus, some works indicate that the photometric method can be successfully used for research and is almost not inferior to traditional methods when it is necessary to use a centimeter tape [17, 21].

Regarding teleroentgenographic measurements, there are also discussions about the choice of the best cephalometric method of research. In the work of Paranhos L. R. and co-authors [18], the use of the SN-GoGn angle is preferred according to the results.

Similar data were obtained by Brazilian researchers during the analysis of 95 teleroentgenograms of 54 men and 41 women aged 15-21 years. Kappa was considered valid for the cephalometric evaluation method for Jarabak SN-GoGn (0.06 and $36.8 \%$ ) [9].

It is obvious that the question of studying the relationship between cephalometric indicators, face type, odontometric indicators has not yet been resolved. Work on data collection for representatives of different ethnic groups continues in different parts of the world [11, 23, 25].

Given the above data, there is a need to conduct a study of cephalometric parameters in the Ukrainian population, taking into account sex, type of bite and face type.

The aim of the study was to establish the characteristics of cephalometric parameters in Ukrainian young men and young women with orthognathic occlusion, which usually do not change during surgical and orthodontic treatment, depending on sex and facial types according to Garson.

\section{Materials and methods}

On the basis of the clinic "Winintermed" teleroentgenography was performed using a dental conebeam tomograph Veraviewepocs 3D Morita (Japan) in the mode of cephalometric examination of 8 young men (aged 17 to 21 years) and 17 young women (aged 16 to 20 years) who had physiological bite as close as possible to the orthognathic (hereinafter orthognathic) which is determined by 11 points according to Bushan M. G. and others [3]. Cephalometric analysis was performed using OnyxCeph ${ }^{3 \text { тм }}$ software, 3DPro version, Image Instruments $\mathrm{GmbH}$, Germany (№ URSQ-1799 software license). From the database of the research center of National Pirogov Memorial Medical University, Vinnytsya selected 38 young men and 55 young women of the same age with orthognathic occlusion, who also underwent teleroentgenography followed by cephalometric analysis.

Committee on Bioethics of National Pirogov Memorial Medical University, Vinnytsya (protocol № 9 from 21.11.2019) it was established that the conducted research meets the bioethical and moral and legal requirements of the Declaration of Helsinki, the Convention of the Council of Europe on human rights and biomedicine (1977), the relevant provisions of the $\mathrm{WHO}$ and the laws of Ukraine according to the order of the Ministry of Health of Ukraine № 281 from 01.11.2000.

The type of face was determined according to the morphological index of Garson (Fig. 1) according to the 


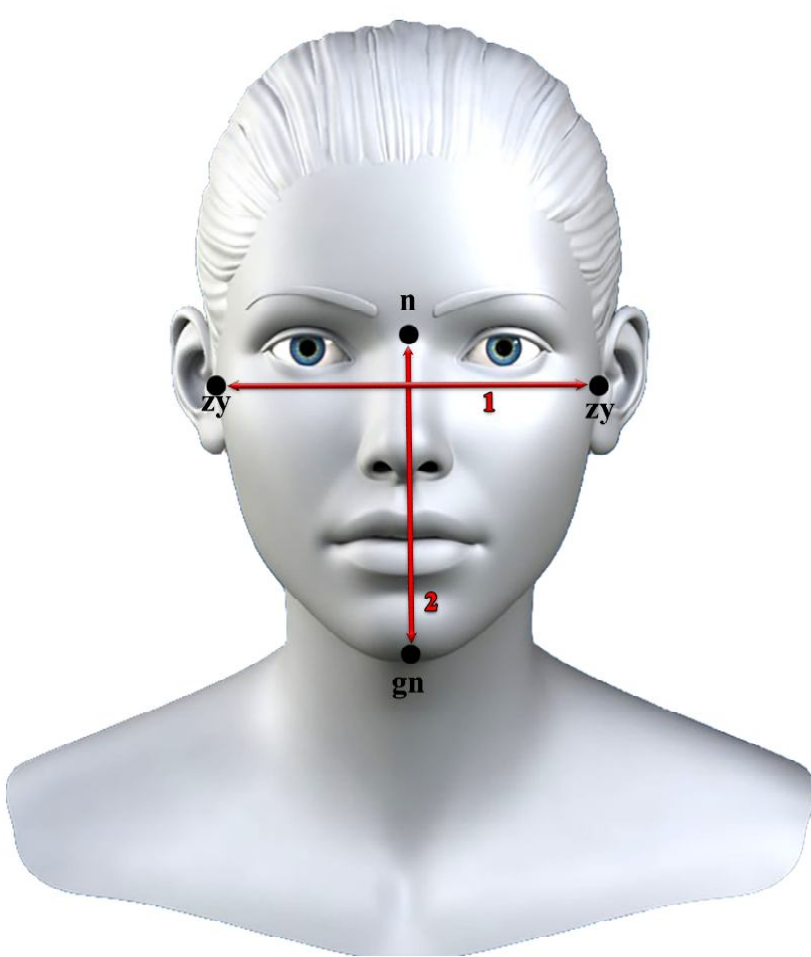

Fig. 1. Basic cephalometric points and measurements to determine the type of face according to the morphological index of Garson. 1 (zy_zy) - the largest width of the face (distance between the cheekbones); $\mathbf{2}$ (n_gn) - morphological length of the face (direct distance from the point nasion to the point gnathion).

appropriate formula [19]: morphological length of the face / maximum width of the face $\times 100$.

When the value of the indicator up to 78.9 young men or young women were attributed to group with a very wide face; 79.0-83.9 - with a wide face; 84.0-87.9 - with an average face; 88.0-92.9 - with a narrow face; 93.0 and more - with a very narrow face. The following distribution is established: young men with a very wide face - 5 ; young women with a very wide face - 25 ; young men with a wide face - 22; young women with a wide face - 25; young men with an average face - 11; young women with a medium face - 10; young men with a narrow face - 8; young women with a narrow face - 12 .

The main cephalometric points and measurements are basic in modern cephalometric analyzes and are included in the parameters that usually do not change during surgical and orthodontic treatment are presented in Figure 2.

Estimated indicators are calculated on the basis of those shown in Figure 2: N-S:S-Ar' - the ratio of the distances S-Ar' (12) and N-S (2) in the cephalometric analysis of Bjork; S-ar:ar-Go - ratio of the distances S-Ar (5) and Ar-Go (7) in the Roth-Jarabak cephalometric analysis.

Statistical analysis of teleroentgenographic indicators was performed in the license package "Statistica 6.0" using non-parametric methods of evaluation of the obtained results.

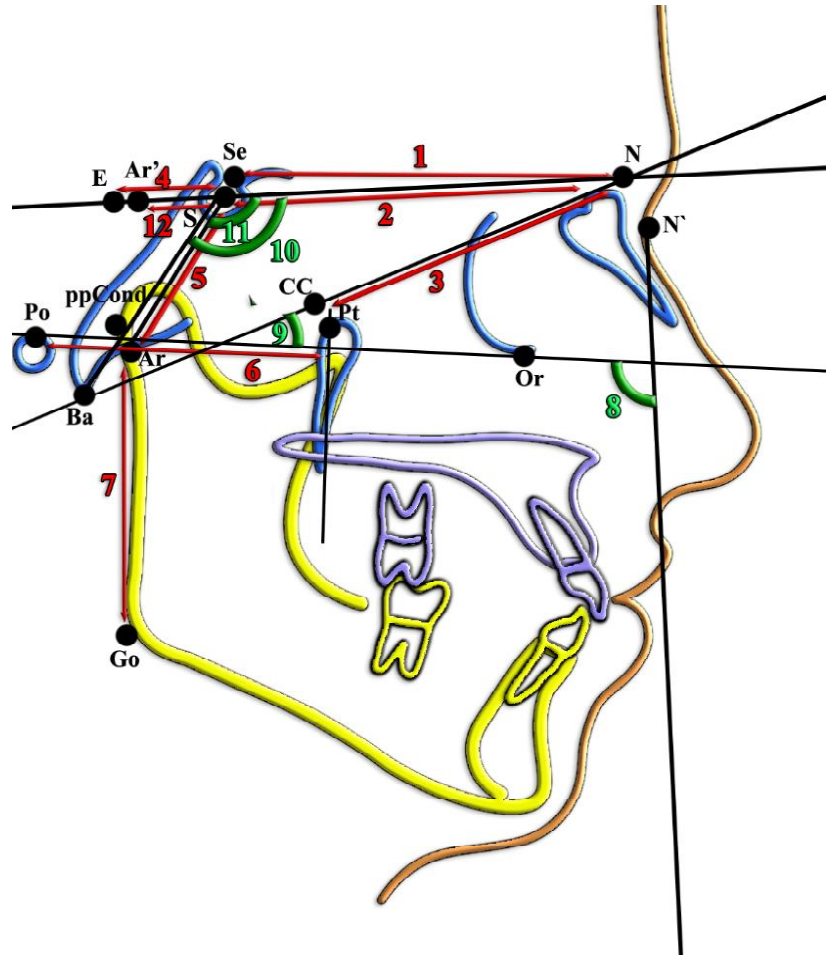

Fig. 2. Basic cephalometric points and measurements that usually do not change during surgical and orthodontic treatment. 1 (N-Se) - (length of the front part of the skull base according to Schwartz, distance Se-N) - distance from the point Se to the point $\mathbf{N}(\mathrm{mm}) ; \mathbf{2}$ (N-S) - (length of the front part of the base of the skull according to Roth-Jarabak) - the distance from point $\mathbf{N}$ to point $\mathbf{S}(\mathrm{mm}) ; \mathbf{3}(\mathbf{N}$ CC) - (anterior length of the base of the skull according to Ricketts) - the distance from the point $\mathbf{N}$ to the point CC (mm); 4 (S-E) (length of the back of the skull base according to Steiner, distance $\mathrm{S}-\mathrm{E})$ - the distance from point $\mathbf{S}$ to the structural point $\mathbf{E}$, which is located at the intersection of the perpendicular drawn from the point ppCond to the line S-N (mm); $\mathbf{5}$ (S-Ar) - (length of the lateral cranial base according to Roth-Jarabak) - the distance from the point $\mathbf{S}$ to the point $\mathbf{A r}(\mathrm{mm}) ; \mathbf{6}$ (P-PTV) - (distance P-PTV according to Ricketts) - the distance from point Po to point $\mathbf{P t}$, determined parallel to the Frankfurt plane (mm); 7 (Ar-Go) - (length of the branch of the lower jaw according to Burstone) - the distance from the point $\mathbf{A r}$ to the point $\mathbf{t G o}(\mathrm{mm}) ; \mathbf{8}(\mathbf{H})$ - $(\mathrm{H}$-angle according to Schwartz) - the angle formed by the lines Po-Or (Frankfurt plane (Fp)) and Pn (nasal perpendicular, perpendicular to the line from the point $\mathbf{N}$ 'to the line $\mathbf{S e}-\mathbf{N}\left({ }^{\circ}\right)$; 9 (POr -NBa) - (Ricketts cranial deflection angle) - angle formed by Po-Or and $\mathbf{B a - N}$ lines ( ${ }^{\circ}$ ); 10 (NS-Ba) - (NS-Ba angle according to Bjork) - angle formed by $\mathbf{N}-\mathbf{S}$ and $\mathbf{S}-\mathrm{Ba}$ lines $\left({ }^{\circ}\right)$; 11 (NS-Ar) - (saddle angle according to Bjork) - angle formed by lines N-S and S-Ar $\left({ }^{\circ}\right.$ ); 12 S-Ar' - (distance of Bjork joint location) - distance from point $\mathbf{S}$ to point $\mathbf{A r}$.

\section{Results}

The percentage range of cephalometric parameters, which usually do not change during surgical and orthodontic treatment depending on the facial types of Ukrainian young men and young women with orthognathic occlusion are shown in Table 1.

When comparing the value of the distance $\mathbf{N}-\mathbf{S}$ (according to Jarabak) in young men with very wide 
Table 1. Percentage range (25.0th - 75.0th percentl) of cephalometric parameters, which usually do not change during surgical and orthodontic treatment depending on the facial types of Ukrainian young men and young women.

\begin{tabular}{|c|c|c|c|c|c|}
\hline \multirow{2}{*}{ Indicator } & \multirow{2}{*}{ Sex } & \multicolumn{4}{|c|}{ Face type } \\
\hline & & very wide & wide & average & narrow \\
\hline \multirow{2}{*}{ Angle N-S-Ar $\left(^{\circ}\right)$} & M & $124.8-130.5$ & $121.8-129.0$ & $120.9-129.4$ & $119.5-129.9$ \\
\hline & W & $120.2-129.6$ & $121.4-128.4$ & $123.9-129.5$ & $120.9-129.2$ \\
\hline \multirow{2}{*}{ Angle N-S-Ba $\left({ }^{\circ}\right)$} & M & $129.6-132.9$ & $126.6-132.5$ & $124.7-133.8$ & $123.2-132.3$ \\
\hline & W & $125.1-133.3$ & $125.6-134.8$ & $128.9-135.6$ & $126.4-133.2$ \\
\hline \multirow{2}{*}{ Ratio N-S:S-Ar' } & $\mathrm{M}$ & $3.2-3.6$ & $3.1-4.1$ & $3.3-4.2$ & $3.0-4.05$ \\
\hline & W & $3.4-4.3$ & $3.5-3.9$ & $3.1-3.8$ & $3.4-4.0$ \\
\hline \multirow{2}{*}{ Distance N-S (mm) } & M & $70.0-13.5$ & $12.8-13.5$ & $12.8-13.5$ & $12.8-13.5$ \\
\hline & W & $12.8-13.5$ & $12.8-13.5$ & $12.8-13.5$ & $12.8-13.5$ \\
\hline \multirow{2}{*}{ Distance N-S (mm) } & M & $31.0-36.0$ & $33.0-37.0$ & $33.0-38.0$ & $35.0-37.5$ \\
\hline & W & $31.0-35.0$ & $31.0-34.0$ & $30.0-34.0$ & $30.5-33.0$ \\
\hline \multirow{2}{*}{ Ratio S-ar:ar-Go } & M & $54.0-69.0$ & $59.0-73.0$ & $60.0-72.0$ & $64.5-71.0$ \\
\hline & W & $62.0-75.0$ & $64.0-76.0$ & $60.0-71.0$ & $61.0-71.0$ \\
\hline \multirow{2}{*}{ Distance Ar-Go (mm) } & M & $55.1-57.9$ & $50.1-55.4$ & $49.6-55.4$ & $50.9-55.3$ \\
\hline & W & $44.3-49.4$ & $44.0-49.1$ & $43.3-50.7$ & $45.7-49.0$ \\
\hline \multirow{2}{*}{ Distance N-Se (mm) } & M & $69.0-70.0$ & $67.0-70.0$ & $68.0-71.0$ & $67.0-72.5$ \\
\hline & W & $63.0-66.0$ & $62.5-67.5$ & $63.0-66.0$ & $63.0-68.0$ \\
\hline \multirow{2}{*}{ Angle $H^{\circ}$ ) } & M & $93.0-98.0$ & $92.0-97.0$ & $94.0-96.0$ & $91.0-95.0$ \\
\hline & W & $92.0-96.0$ & $93.0-95.0$ & $93.0-98.0$ & $92.0-96.5$ \\
\hline \multirow{2}{*}{ Distance S-E (mm) } & M & $20.0-22.0$ & $18.0-23.0$ & $18.0-22.0$ & $19.0-23.5$ \\
\hline & W & $16.0-20.0$ & $18.0-21.0$ & $18.0-22.0$ & $16.5-19.0$ \\
\hline \multirow{2}{*}{ Angle Por-NBa $\left({ }^{\circ}\right)$} & M & $23.3-25.2$ & $23.7-27.7$ & $23.7-28.9$ & $23.3-26.5$ \\
\hline & W & $23.4-26.2$ & $25.3-27.5$ & $24.7-28.1$ & $24.4-26.8$ \\
\hline \multirow{2}{*}{ Distance N-CC (mm) } & M & $56.5-59.7$ & $56.6-59.8$ & $55.5-58.9$ & $57.7-60.3$ \\
\hline & W & $52.7-57.3$ & $51.1-57.1$ & $53.5-54.6$ & $51.5-55.5$ \\
\hline \multirow{2}{*}{ Distance P-PTV $(\mathrm{mm})$} & M & $-41.1--38.4$ & $-42.3--38.2$ & $-42.1--36.5$ & $-41.7--38.9$ \\
\hline & W & $-38.9--34.9$ & $-39.7--36.5$ & $-39.7--34.9$ & $-39.0--33.6$ \\
\hline
\end{tabular}

Notes: $\mathrm{M}$ - young men; $\mathrm{W}$ - young women.

$(70.40 \pm 2.19 \mathrm{~mm})$, wide $(70.41 \pm 2.50 \mathrm{~mm})$, medium $(76.09 \pm 19.65 \mathrm{~mm})$ and narrow $(71.38 \pm 4.90 \mathrm{~mm})$ face types are established reliably $(p<0.05-0.01)$ higher values compared to young women of the corresponding type of face (respectively $67.92 \pm 6.99 \mathrm{~mm}-67.32 \pm 5.06 \mathrm{~mm}$ $66.10 \pm 2.33 \mathrm{~mm}-66.08 \pm 3.34 \mathrm{~mm})$.

The value of the S-Ar distance (according to Jarabak) in young men with wide $(34.95 \pm 2.80 \mathrm{~mm})$ and narrow $(37.13 \pm 4.26 \mathrm{~mm})$ face types is significantly $(p<0.01-0.001)$ greater than in young women of the corresponding face type (respectively $32.72 \pm 3.14 \mathrm{~mm}-31.50 \pm 2.02 \mathrm{~mm}$ ).

When comparing the value of the distance Ar-Go (according to Burstone) in young men with very wide $(56.38 \pm 2.80 \mathrm{~mm})$, wide $(52.67 \pm 5.13 \mathrm{~mm})$, medium $(56.11 \pm 16.53 \mathrm{~mm})$ and narrow $(53.59 \pm 3.37 \mathrm{~mm})$ face types are established reliably $(p<0.05-0.001)$ higher values compared to young women of the corresponding type of face (respectively $47.97 \pm 5.89 \mathrm{~mm}-47.20 \pm 6.46 \mathrm{~mm}$ $46.69 \pm 4.27 \mathrm{~mm}-47.61 \pm 3.50 \mathrm{~mm}$ ). Also, in young men with a very wide face, the value of this indicator tends $(p=0.066)$ to higher values than in young men with a wide face.

The value of the distance $\mathbf{N}-\mathrm{Se}$ (according to Schwarz) in young men with very wide $(68.80 \pm 2.95 \mathrm{~mm})$, wide $(68.95 \pm 2.73 \mathrm{~mm})$, medium $(74.64 \pm 19.46 \mathrm{~mm})$ and narrow $(70.50 \pm 4.96 \mathrm{~mm})$ facial types significantly $(p<0.05-0.001)$ is greater compared to young women of the corresponding type of face (respectively $66.28 \pm 6.78 \mathrm{~mm}-65.42 \pm 5.37 \mathrm{~mm}$ - $64.70 \pm 2.31 \mathrm{~mm}-65.50 \pm 3.29 \mathrm{~mm}$ ).

When comparing the value of the distance S-E (according to Steiner) found only a tendency $(p=0.054)$ to higher values of this indicator in young men with a narrow 
$(21.50 \pm 3.25 \mathrm{~mm})$ face type compared to young women with a narrow face type $(18.08 \pm 3.53)$.

When comparing the value of the angle POr-NBa (according to Ricketts) found only a tendency $(p=0.074)$ to higher values of this indicator in young women with medium face type $(26.76 \pm 1.95 \mathrm{~mm})$ compared to young women with very wide face type $(25.18 \pm 2.34 \mathrm{~mm})$.

The value of the distance N-CC (according to Ricketts) in young men with very wide $(58.56 \pm 2.24 \mathrm{~mm})$, wide $(57.59$ $\pm 3.28 \mathrm{~mm})$, medium $(57.39 \pm 2.71 \mathrm{~mm})$ and narrow $(59.03 \pm 2.36 \mathrm{~mm})$ facial types significantly $(p<0.05-0.01)$ is greater compared to young women of the corresponding face type (respectively $56.22 \pm 6.22 \mathrm{~mm}-54.45 \pm 3.72 \mathrm{~mm}$ $54.37 \pm 2.86 \mathrm{~mm}-53.76 \pm 3.05 \mathrm{~mm}$ ).

When comparing the P-PTV distance (according to Ricketts) in young men with very wide $(-40.18 \pm 2.48 \mathrm{~mm})$, wide $(-40.17 \pm 2.50 \mathrm{~mm})$ and narrow $(-40.01 \pm 3.85 \mathrm{~mm})$ facial types, it was established reliably $(p<0.05-0.01)$ higher values of this indicator compared to young women of the corresponding type of face (respectively $-37.22 \pm 4.16 \mathrm{~mm}$ $-38.01 \pm 3.68 \mathrm{~mm}--36.94 \pm 3.32 \mathrm{~mm}$ ).

There are no significant or trend differences in the magnitude of the angles N-S-Ar (according to Bjork), N-SBa (according to Bjork) and $\mathrm{H}$ (according to Schwartz), as well as the ratios N-S:S-Ar' (according to Bjork) and Sar:ar-Go (according to Jarabak) both between young men and young women of the corresponding face types, and between representatives of the corresponding sex of different face types.

\section{Discussion}

Thus, as a result of our research, pronounced manifestations of sexual dimorphism of only linear cephalometric parameters were established, which usually do not change during surgical and orthodontic treatment. Thus, young men with different face types have significantly greater values of N-S, Ar-Go, N-Se, N-CC, P-PTV and S-Ar distances than young women of the corresponding face types. For the magnitude of angles and ratios of cephalometric parameters, which usually do not change during surgical and orthodontic treatment, no manifestations of sexual dimorphism of these indicators were found between young men and young women of the corresponding face types. Also, we have not found significant or trends in the differences of these parameters between young men or between young women with different face types.

A group of authors led by Correa S. [4] found that most sizes of the branches of the mandible are the largest in brachycephalic $(p<0.0001)$.

In a study conducted on ethnic Lebanese, it was found that men have higher upper and lower jaws parameters,

\section{References}

[1] Abdelali, H., Benyahia, H., Abouqal, R., Azaroual, M. F., \& Zaoui, F. (2012). Associations between alveolar heights and vertical skeletal pattern in Moroccan adults: a cephalometric study of while the ANB angle is higher in women. Lebanese women also have a more convex facial profile than men.

Teleroentgenograms of 46 men and 19 women aged 18-25 years, ethnic residents of Yemen, without orthodontic treatment in the anamnesis were studied and the data were statistically processed. It was found that Yemenis have higher SNA, SNB and SNPg angles [5].

The results of a study by Kolte R. A. and others [14] revealed that there are significant differences between the facial index, lip size and type of periodontium. For the most part, close correlations have been found between the thickness of the gums and the inclination of the front teeth of the mandible.

Laranjo F. and Pinho T. [15] studied the relationship between cephalometric and odontometric parameters in people with open bite. It was found that persons with an open bite have increased dental alveolar height of the upper and lower jaws and anterior height of the face.

Facial index scores were obtained for 33 people with a complete set of teeth and for 33 people with adentia. Also, each of the subjects underwent teleroentgenography to measure OP, FHP, CP, OP-FHP and OP-CP. After statistical processing, a positive correlation was found between TFI and OP-FHP, and a negative correlation for OP-FHP and OP-CP in individuals with a complete set of teeth [20].

44 young men of Ukrainian origin underwent a cephalometric, odontometric study with subsequent determination of the type of face in accordance with Garson's index. Statistical analysis of the data was used to develop regression models needed to construct correct dental arches. A total of 18 reliable models were built with a coefficient from 0.645 to 0.944 [16].

Summing up, it is safe to say that the study consistently complements and confirms the results of scientific work of other domestic and foreign researchers.

\section{Conclusions}

1. In Ukrainian young men and young women with orthognathic occlusion and different types of faces according to Garson, the percentile range of cephalometric parameters has been established, which usually do not change during surgical and orthodontic treatment.

2. The expressed manifestations of sexual dimorphism of cephalometric parameters which usually do not change during surgical and orthodontic treatment (bigger values at young men) are established only for the majority of the linear sizes at representatives of various types of face.

3. For cephalometric parameters, which usually do not change during surgical and orthodontic treatment in both young men and young women, there are almost no significant or tendencies of differences between representatives with different face types according to Garson.

127 clinical cases. International orthodontics, 10(1), 43-53. doi: 10.1016/j.ortho.2011.09.001

[2] Ahsan, A., Yamaki, M., Hossain, Z., \& Saito, I. (2013). Craniofacial 
cephalometric analysis of Bangladeshi and Japanese adults with normal occlusion and balanced faces: A comparative study. Journal of orthodontic science, 2(1), 7-15. doi: 10.4103/ 2278-0203.110327

[3] Bushan, M. H., Vasylenko, Z. S., Hryhoreva, L. P. (1990). Cправочник по ортодонти [Handbook of orthodontics]. Кишенев: Картя Молдовеняскэ - Kishinev: Kartia Moldoveniaske.

[4] Correa, S., Lopes Motta, R. H., Silva, M. B. F., Figueroba, S. R., Groppo, F. C., \& Ramacciato, J. C. (2019). Position of the Mandibular Foramen in Different Facial Shapes Assessed by Cone-Beam Computed Tomography-A Cross-Sectional Retrospective Study. The Open Dentistry Journal, 13(1), 544550. doi: 10.2174/1874210601913010544

[5] Daer, A. A., \& Abuaffan, A. H. (2016). Skeletal and dentoalveolar cephalometric features of anterior open bite among Yemeni adults. Scientifica, 3147972. doi: 10.1155/2016/3147972

[6] Daraze, A., Delatte, M., Saba, S. B., \& Majzoub, Z. (2017). Craniofacial characteristics in the sagittal dimension: a cephalometric study in Lebanese young adults. International orthodontics, 15(1), 114-130. doi: 10.1016/j.ortho.2016.12.001

[7] Darkwah, W. K., Kadri, A., Adormaa, B. B., \& Aidoo, G. (2018). Cephalometric study of the relationship between facial morphology and ethnicity. Translational Research in Anatomy, 12, 20-24. doi: 10.1016/j.tria.2018.07.001

[8] Davalos, G. E. A., Lozano, M. B., \& Sosa, G. O. (2013). Cephalometric description of facial patterns in skeletal open bite. Revista Odontologica Mexicana, 17(1), 15-19.

[9] de Novaes Benedicto, E., Kairalla, S. A., Oliveira, G. M. S., Junior, L. R. M., Rosario, H. D., \& Paranhos, L. R. (2016). Determination of vertical characteristics with different cephalometric measurements. European journal of dentistry, 10(1), 116-120. doi: 10.4103/1305-7456.175694

[10] Dmitriev, M., Gunas, V., Polishchuk, S., Olkhova, I., \& Kumar, A. (2020). Modeling of Central Incisors Position Indicators in boys and girls according to CC. Steiner method for Forensic Dental Identification. J Indian Acad Forensic Med, 42(3), 155-160. doi: 10.5958/0974-0848.2020.00043.3

[11] Doni, R. P. K., Janaki, C. S. \& Vijayaraghavan, V. (2013). A study on measurement and correlation of cephalic and facial indices in males of South Indian population. International Journal of Medical Research \& Health Sciences, 2(3), 439446. doi: 10.5958/j.2319-5886.2.3.076

[12] Gunas, V. I., Kotsyura, O. O., Babych, L. V., Shevchuk, Y. G., \& Cherkasova, O. V. (2020). Features correlations of the sizes of molars with cephalometric indicators of men of the western region of Ukraine. Reports of Morphology, 26(2), 51-61. doi: 10.31393/morphology-journal-2020-26(2)-08

[13] Han, S. H., Lee, E. H., Cho, J. H., Chae, J. M., Kim, S. C., Chang, N. Y., \& Kang, K. H. (2013). Evaluation of the relationship between upper incisor exposure and cephalometric variables in Korean young adults. Korean journal of orthodontics, 43(5), 225-234. doi: 10.4041/kjod.2013.43.5.225

[14] Kolte, R. A., Kolte, A. P., Kharkar, V. V., \& Bawankar, P. (2020). Influence of facial index, facial profile, lip size, and angulations of teeth on gingival characteristics of anterior teeth: A genderbased evaluation. Journal of Esthetic and Restorative

\section{Dentistry, 32(5), 496-504. doi: 10.1111/jerd.12600}

[15] Laranjo, F., \& Pinho, T. (2014). Cephalometric study of the upper airways and dentoalveolar height in open bite patients. International orthodontics, 12(4), 467-482. doi: 10.1016/ j.ortho.2014.10.005

[16] Marchenko, A. V., Shinkaruk-Dykovytska, M. M., Pozur, T. P., Gunas, V. I., \& Orlovskiy, V. O. (2020). Models of individual linear dimensions necessary for the construction of the correct form of dental arches in young men with a wide face, depending on the features of odontometric and cephalometric indicators. Wiad Lek, 73(6), 1103-1107. doi: 10.36740/WLek202006104

[17] Nicoo, M., Fakhri, F., Nikou, F., \& Parastesh, A. (2019). Correlation Between Cephalometric and Photographic Results of Determining the Lower Anterior Facial Height. Hormozgan Medical Journal, 23(1), e86932. doi: 10.5812/hmj.86932

[18] Paranhos, L. R., Benedicto, E. N., Nunes, M. F., Kairalla, S. A., Furquim Siqueira, D., \& Cesar Torres, F. (2012). Correlation of different cephalometric measurements to define facial type. International Journal of Orthodontics, 23(1), 31-37. PMID: 22533026

[19] Profit, W. R. (2017). Современная ортодонтия (4-е изд., пер. с англ.) [Modern orthodontics (4th ed., trans. from English)]. М.: МЕДпресс-информ - М.: MEDpress-inform.

[20] Rajawat, I., Venkataramana, V., Patil, P., Guram, G., Gupta, N., Lau, M., ... \& Shah, D. M. (2014). A cephalometric evaluation for co-relation of different facial types with occlusal plane in dentulous and edentulous patients. OHDM, 13(4), 1188-1191.

[21] Raval, K., Patel, R., Mehta, F., Paria, P., Parekh, H., \& Trivedi, R. (2020). Correlation of Transverse Facial Dimension in Vertical Facial Morphology-True Size Frontal Face Photographic Study. Journal of Dental and Medical Sciences, 19(2), 50-60. doi: 10.9790/0853-1902055060

[22] Shah, T., Thaker, M. B., \& Menon, S. K. (2015). Assessment of cephalic and facial indices: a proof for ethnic and sexual dimorphism. J Forensic Sci Criminol, 2(4), 101. doi: 10.15744/ 2348-9804.2.401

[23] Singh, S., Mehta, K., Sandhu, N., \& Sharma, A. (2016). An anthropometric and cephalometric study to correlate facial form to pharyngeal airway in Class I and Class II malocclusions. Journal of Indian Orthodontic Society, 50(2), 83-93. doi: 10.4103/0301-5742.179946

[24] Sobon, J. S., Cherkasova, O. V., Gunas, V. I., Babych, L. V., \& Kotsyura, O. O. (2020). Correlations of linear sizes of molars with cephalometric indicators of practically healthy men of the southern region of Ukraine. Biomedical and Biosocial Anthropology, (38), 36-46. doi: 10.31393/bba38-2020-06

[25] Trivedi, H., Azam, A., Tandon, R., Chandra, P., Kulshrestha, R., \& Gupta, A. (2017). Correlation between morphological facial index and canine relationship in adults - An anthropometric study. Journal of Orofacial Sciences, 9(1), 16-21. doi: 10.4103/ jofs.jofs_50_16

[26] Yamauchi, T., Kimura, R., Kawaguchi, A., Sato, T., Yamaguchi, K., Toma, T., ... \& Ishida, H. (2016). A comparative study of craniofacial measurements between Ryukyuan and mainland Japanese females using lateral cephalometric images. Anthropological Science, 151206. doi: 10.1537/ase.151206 